\title{
Classification of the Coefficients of Variation of Parameters Evaluated in Japanese Quail Experiments
}

ISSN 1516-635X Apr - Jun 2014 / v.16 / n.2 / 97-100

http://dx.doi.org/10.1590/1516-635x160297-100

\section{-Author(s)}

Leal DHV

Faria Filho DE de

Oliveira EMB"
Zootecnista.
Departamento de Zootecnia, Universidade de São Paulo, Faculdade de Zootecnia En- genharia de Alimentos, Pirassununga, São Paulo, Brasil
III Instituto de Ciências Agrárias da Universi- dade Federal de Minas Gerais, Montes Cla- ros, Minas Gerais, Brasil.

\section{ABSTRACT}

The objective of this study was to design a classification range of the coefficients of variation (CV) of traits used in experiments with eggtype Japanese quails (Coturnix coturnix japonica). The journal Revista Brasileira de Zootecnia was systematically reviewed, using the key word 'quail' during the period of January, 2000 to 2010. The CV of feed intake (g/bird/d), egg production (\%/bird/d), egg weight (g), egg mass (g/bird/d), feed conversion ratio per dozen eggs (g/dozen), feed conversion ratio per egg mass $(\mathrm{g} / \mathrm{g})$, and egg specific gravity $(\mathrm{g} / \mathrm{mL})$ were collected. For each parameter, CV were classified using the following median (MD) and pseudo-sigma (PS) ratio as follows: low (CV $\leq \mathrm{MD}-$ $P S)$, medium (MD - PS $<C V \leq M D+P S)$, high ( $M D+P S<C V \leq M D+$ $2 P S)$ and very high $(C V>M D+2 P S)$. According to the results, it was concluded that each parameter has a specific classification range that should be taken into account when evaluating experimental precision.

\section{INTRODUCTION}

The coefficient of variation is obtained by the residual standard deviation as a percentage of the general mean of the experiment. Therefore, it measures experimental precision because the residual standard deviation is the square root of residual mean square, which contains all the variation due to experimental error. In addition, the CV allows comparing experiments, independently of the unit used.

In experiments with Japanese quails, there are reference values of specific CV that identify classification ranges relative to their degree of precision, as it happens in pig production (Judice et al., 1999), beef cattle (Judice et al., 2002), equine nutrition (Lana et al., 2006), broilers (Mohallem et al., 2008), and commercial layers (Faria Filho et al., 2010).

Therefore, the distribution of $C V$ values in experiments with Japanese quails need to be studied, as most researchers have compared their results with the classification suggested by Gomes (2000) as low, when CV values are lower than 10\%; medium, with CV values between 10 and $20 \%$, high, with CV value between 20 and 30\%, and very high, when CV values are higher than $30 \%$.

Amaral etal. (1997) and Judice et al. (1999) suggest that the normality of $\mathrm{CV}$ distribution should be determined to establish variability ranges. However, working with upland rice crops, Costa et al. (2002) presented a new method of CV classification that can be applied with data do not have normal distribution. This method is based on the use of median and pseudo-sigma, instead of mean and standard deviation.

In order to obtain a consistent compilation of published studies, the method of systematic literature review can be used (Castro et al. 2002). This is a scientific technique to review literature, using specific methods to identify, select, and critically analyze relevant studies. Therefore, 
search strategy, databases, period of review, and criteria for the inclusion of studies must be previously defined. All these aspects should be mentioned in the text. Excluded articles must be mentioned and their exclusion justified. This will render these systematic reviews reproducible and, in general, unbiased.

This study aimed at verifying if the parameters evaluated in experiments with Japanese quails (Coturnix coturnix japonica) have specific classification ranges of the coefficient of variation by using the method of systematic literature review for data collection and the method of Costa et al. (2002) to classify the CV.

\section{MATERIALS AND METHODS}

The systematic literature review (Castro et al., 2002) was carried out using the journal Revista Brasileira de Zootecnia of issues published between January, 2000 and January, 2010. The website of Sociedade Brasileira de Zootecnia (Brazilian Society of Animal Science) was accessed (www.sbz.org.br; access in April, 2010) and the path revista $\rightarrow$ revista on-line $\rightarrow$ entrar $\rightarrow$ consulta por palavra-chave (journal $\rightarrow$ online journal $\rightarrow$ enter $\rightarrow$ search key word) was followed. The key word used was "quail" to search in Portuguese the fields title, key words, and abstract.

Studies with egg-type Japanese quails (Coturnix coturnix japonica) during lay and presenting $\mathrm{CV}$ for at least one of the following parameters were selected: feed intake $(\mathrm{g} / \mathrm{bird} / \mathrm{d})$, egg production $(\% / \mathrm{bird} / \mathrm{d})$, egg weight (g), egg mass ( $g /$ bird/d), feed conversion ratio per dozen eggs ( $g /$ dozen), feed conversion ratio per egg mass $(\mathrm{g} / \mathrm{g})$, and egg specific gravity $(\mathrm{g} / \mathrm{mL})$.

The number of observations, minimum and maximum values, mean and standard deviation were calculated. The median (MD) was calculated according the equation: $M D=(Q 1+Q 3) / 2$, where $Q 1$ is the first quartile and Q3 is the third quartile. pseudo-sigma (PS) was calculated according to the equation: $\mathrm{PS}=(\mathrm{Q} 3-$ Q1)/1.35 (Tukey, 1977). Data normality was evaluated by the Cramer-Von-Mises test. Analyses were carried out using SAS ${ }^{\circledR}$ software package (Littell et al., 2002).

$C V$ ranges were determined according to the methodology proposed by Costa et al. (2002) as a function of $\mathrm{MD}$ and $\mathrm{PS}$. The following $\mathrm{CV}$ ranges were determined: low (CV $\leq \mathrm{MD}-\mathrm{PS})$, medium (MD - PS < $C V \leq M D+P S)$, high (MD + PS $<C V \leq M D+2 P S)$, and very high $(C V>M D+2 P S)$.

\section{RESULTS AND DISCUSSION}

The systematic literature review found 41 articles, out of which 24 were selected: Araújo et al. (2007), Barreto et al. 2007 a, 2007 b, 2007 c, 2007 d), Costa et al. (2007), Costa et al. (2008a, 2008 b), Costa et al. (2009), Freitas et al. (2005), Mori et al. (2005), Moura et al. (2008), Murakami et al. (2006), Oliveira et al. (2009), Pinheiro et al. (2008), Pinto et al. (2002), Pinto et al. (2003 a, 2003 b), Ribeiro et al. (2003), Rizzo et al. (2008), Silva et al. (2002), Silva et al. (2003), Soares et al. (2007), and Umigi et al. (2007).

The number of observations, minimum and maximum values, mean, standard deviation, median, pseudo-sigma, and Cramer-Von-Mises test results of the CV values of the evaluated parameters are presented in Table 1. Only feed intake CV values presented normal distribution, and therefore the methodology proposed by Costa et al. (2002) was applied to establish CV classification ranges because it does not assume data normality.

Table 2 shows the CV classification ranges for the main parameters used in Japanese quail experiments.

The parameter egg specific gravity is highlighted as its classification range is very narrow, which indicated it is a very stable parameter. The same behavior of egg specific gravity results was found by Faria Filho et

Table 1 - Number of observations (N), minimum value (Min), maximum value (Max), mean (X), standard deviation (SD), median (Me), pseudo-sigma (PS), and Cramer-Von-Mises normality test of feed intake (FI), egg production (EP), egg weight (EW), egg mass (EM), feed conversion ratio per dozen eggs (FCRDz), feed conversion ratio per egg mass (FCREM), and egg specific gravity (ESG) of egg-type Japanese quails.

\begin{tabular}{|c|c|c|c|c|c|c|c|c|}
\hline Parameter & $\mathrm{N}$ & Min & Max & Mean & SD & $\mathrm{Me}$ & PS & Normality \\
\hline $\mathrm{FI}$ & 24 & 2.48 & 11.19 & 5.23 & 2.12 & 5.08 & 2.25 & $0.08 \mathrm{~ns}$ \\
\hline EP & 25 & 2.50 & 16.23 & 6.93 & 3.68 & 7.13 & 3.55 & $0.15^{\star}$ \\
\hline EW & 23 & 1.37 & 6.22 & 3.17 & 1.06 & 2.95 & 0.78 & $0.13^{*}$ \\
\hline EM & 23 & 3.50 & 15.81 & 8.02 & 3.62 & 7.34 & 3.61 & $0.15^{*}$ \\
\hline FCRDz & 19 & 3.30 & 34.67 & 8.31 & 7.01 & 6.74 & 3.14 & $0.37 * *$ \\
\hline FCREM & 22 & 3.17 & 39.96 & 9.18 & 7.95 & 7.54 & 4.20 & 0.36 ** \\
\hline ESG & 8 & 0.09 & 2.06 & 0.47 & 0.69 & 0.33 & 0.30 & $0.27 * *$ \\
\hline
\end{tabular}

ns $=$ not significant, ${ }^{*} p<0.05,{ }^{*}{ }^{*} p<0.01$ 
Table 2 - Coefficient of variation ranges (CV, \%) of feed intake (FI), egg production (EP), egg weight (EW), egg mass (EM), feed conversion ratio per dozen eggs (FCRDz), feed conversion ratio per egg mass (FCREM), and egg specific gravity (ESG) of egg-type Japanese quails.

\begin{tabular}{llccc}
\hline Parameter & Low & Medium & High & Very high \\
\hline FI & $C V \leq 2.83$ & $2.83<C V \leq 7.33$ & $7.33<C V \leq 9.58$ & $C V \leq 9.58$ \\
EP & $C V \leq 3.58$ & $3.58<C V \leq 10.67$ & $10.67<C V \leq 14.22$ & $C V \leq 14.22$ \\
EW & $C V \leq 2.17$ & $2.17<C V \leq 3.72$ & $3.72<C V \leq 4.50$ & $C V \leq 4.50$ \\
EM & $C V \leq 3.73$ & $3.73<C V \leq 10.94$ & $10.94<C V \leq 14.55$ & $C V \leq 14.55$ \\
FCRDz & $C V \leq 3.60$ & $3.60<C V \leq 9.88$ & $9.88<C V \leq 13.02$ & $C V \leq 13.02$ \\
FCREM & $C V \leq 3.35$ & $3.35<C V \leq 11.72$ & $11.72<C V \leq 15.90$ & $C V \leq 15.90$ \\
ESG & $C V \leq 0.02$ & $0.02<C V \leq 0.63$ & $0.63<C V \leq 0.93$ & $C V \leq 0.93$ \\
\hline
\end{tabular}

al. (2010) in experiments with commercial layers. On the other hand, feed conversion ratio per egg mass presented the widest CV classification range.

The classification of coefficients of variation in the present study should be used as reference by researchers to verify if the CV results obtained, and consequently the precision of their experiments, are within the acceptable range of values. When comparing the classification ranges used in the present study with that proposed by Gomes (2000), in which CV values lower than $10 \%$ are considered low, between 10 and 20\%, medium; between 20 and $30 \%$, high; and higher than $30 \%$ are very high, it is clear that this proposed range does not apply to Japanese quails. For instance, 8\% CV would be considered low in the classification of Gomes (2000), but according to our classification, it is very high for the parameters egg weight and egg specific gravity. This difference may be due to the fact that the proposal of Gomes (2000) is based on agronomic parameters. Moreover, there is considerable variation among animal species, and there are specific classifications for pigs (Judice et al., 1999), beef cattle (Judice et al., 2002), equine nutrition (Lana et al., 2006), broilers (Mohallem et al., 2008), and commercial layers (Faria Filho et al., 2010).

\section{CONCLUSIONS}

It is concluded that there is a specific classification range for each parameter evaluated in egg-type Japanese quails (Coturnix coturnix japonica) that should be taken into account when evaluating experimental precision.

\section{REFERENCES}

Amaral AM, Muniz JA, Souza M. Avaliação do coeficiente de variação como medida da precisão na experimentação com citros. Pesquisa Agropecuária Brasileira 1997; 32 (12): 1221-1225.

Araújo MS, Barreto SLT, Donzele JL, Oliveira RFM, Umigi RT, Oliveira WP, Balbino EM, Assis AP, Maia GUC. Níveis de cromo orgânico na dieta de codornas japonesas mantidas em estresse por calor na fase de postura. Revista Brasileira de Zootecnia 2007;36(3): 584-588.

Barreto SLT, Araujo MS, Umigi RT, Moura WCO, Costa CHR, Sousa MF. Níveis de sódio em dietas para codorna japonesa em pico de postura. Revista Brasileira de Zootecnia 2007a; 36(5):1559-1565.

Barreto SLT, Pereira CA, Umigi RT, Rocha TC, Araujo MS, Silva CS, Torres Filho RA. Determinação da exigência nutricional de cálcio de codornas japonesas na fase inicial do ciclo de produção. Revista Brasileira de Zootecnia 2007 b;36(1):68-78.

Barreto SLT, Quirino BJS, Brito CO, Umigi RT, Araujo MS, Coimbra JSR, Rojas EEG, Freitas JF, Reis RS. Níveis de energia metabolizável para codornas japonesas na fase inicial de postura. Revista Brasileira de Zootecnia 2007 c;36(1):79-85.

Barreto SLT, Quirino BJS, Brito CO, Umigi RT, Araujo MS, Rocha TC, Pereira CG. Efeitos de níveis nutricionais de energia sobre o desempenho e a qualidade de ovos de codornas européias na fase inicial de postura. Revista Brasileira de Zootecnia 2007 d;36(1):86-93.

Castro AA, Saconato H, Guidugli F, Clark OAC. Curso de revisão sistemática e metanálise [accessed: 2005 Nov 10]. São Paulo: LED-DIS/UNIFESP; 2002. Available at: http://www.virtual.epm.br/cursos/metanalise.

Costa CHR, Barreto SLT, Filho RMM, Araujo MS, Umigi RT, Lima HJD. Avaliação do desempenho e da qualidade dos ovos de codornas de corte de dois grupos genéticos. Revista Brasileira de Zootecnia 2008 a;37(10):1823-1828.

Costa CHR, Barreto SLT, Moura WCO, Reis RS, Leite CDS, Maia GVC. Níveis de fósforo e cálcio em dietas para codornas japonesas em postura. Revista Brasileira de Zootecnia 2007;36(6):2037-2046.

Costa FGP, Rodrigues VP, Goulart CC, Junior JGV, Silva JHVS, Souza JG. Nutritional requirements of digestible methionine + cystine for Japanese quails in production phase. Revista Brasileira de Zootecnia 2009;38(12):2389-2393.

Costa FGP, Rodrigues VP, Goulart CC, Neto RCL, Souza JG, Silva JHV. Exigências de lisina digestível para codornas japonesas na fase de postura. Revista Brasileira de Zootecnia 2008 b;37(12):2136-2140.

Costa NHAD, Seraphin JC, Zimmermann FJP. Novo método de classificação de coeficientes de variação para a cultura do arroz de terras altas. Pesquisa Agropecuária Brasileira 2002; 37:243-249.

Faria Filho DE, Dias AN, Veloso ALC, Bueno CFD, Couto FAP, Matos Júnior JB, Barreto KZO, Rodrigues PA, Carneiro WA. Classification of coefficients of variation in experiments with commercial layers. Brazilian Journal of Poultry Science 2010;12(4):215-217.

Freitas AC, Fuentes MFF, Freitas ER, Sucupira FS, Oliveira BCM. Efeito de níveis de proteína bruta e de energia metabolizável na dieta sobre o desempenho de codornas de postura. Revista Brasileira de Zootecnia 2005;34(3):838-846. 
Gomes FP. Curso de estatística experimental. 13 ed. São Paulo: Nobel: 2000.

Judice MG, Muniz JA, Aquino LH, Bearzoti E. Avaliação da precisão experimental em ensaios com bovinos de corte. Ciência e Agrotecnologia 2002;26:1035-1040.

Judice MG, Muniz JA, Carvalheiro R. Avaliação do coeficiente de variação na experimentação com suínos. Ciência e Agrotecnologia 1999;23:170173.

Lana AMQ, Soares Neto J, Almeida FQ, Rezende ASC, Prates RC. Classificação de coeficientes de variação na experimentação com nutrição de eqüinos. Arquivo Brasileiro de Medicina Veterinária e Zootecnia 2006;58:854-859.

Littell RC, Stroup WW, Freund RJ. SAS For Linear Models. 4 ed. Cary: SAS Institute; 2002.

Mohallem DF, Tavares M, Silva PL, Guimarães EC, Freitas RF. Avaliação do coeficiente de variação com medida de precisão em experimentos com frangos de corte. Arquivo Brasileiro de Medicina Veterinária e Zootecnia 2008; 60: 449-453.

Mori C, Garcia EA, Pavan AC, Piccinin A, Scherer MR, Pizzolante CC. Desempenho e qualidade dos ovos de codornas de quatro grupos genéticos. Revista Brasileira de Zootecnia 2005b;34(3):864-869.

Moura GS, Barreto SLT, Donzele JL, Hosoda LR, Pena GM, Angelini MS. Dietas de diferentes densidades energéticas mantendo constante a relação energia metabolizável: nutrientes para codornas japonesas em postura. Revista Brasileira de Zootecnia 2008; 37(9):1628-1633.

Murakami AE, Sakamoto MI, Souza LMG, Franco JRG, Mituo MAO. Determinação do melhor nível de sal comum para codornas japonesas em postura. Revista Brasileira de Zootecnia 2006;36(6):2333-2337.

Oliveira MC, Machado MG, Gonçalves BN, Macedo CMR, Assis FA. Dietas com mananoligossacarídeo e níveis reduzidos de cálcio para codornas japonesas. Revista Brasileira de Zootecnia 2009;38(11):2193-2197.

Pinheiro SRF, Barreto SLT, Albino LFT, Rostagno HS, Umigi RT, Brito CO. Efeito dos níveis de triptofano digestível em dietas para codornas japonesas em postura. Revista Brasileira de Zootecnia 2008;37(6):2012-2016.
Pinto R, Donzele JL, Ferreira AS, Albino LFT, Soares, Silva M A, Pereira TA. Exigência de metionina mais cistina para codornas japonesas em postura. Revista Brasileira de Zootecnia 2003 a;32(5):1166-1173.

Pinto R, Ferreira AS, Albino LFT, Gomes PC, Vargas Júnior JG. Níveis de proteína e energia para codornas japonesas em postura. Revista Brasileira de Zootecnia 2002;31(4):1761-1770

Pinto R, Ferreira AS, Donzele JL, Silva MA, Soares RTRN, Custódio GS, Pena KS. Exigência de Lisina para Codornas Japonesas em Postura. Revista Brasileira de Zootecnia 2003 b; 32(5):1182-1189.

Ribeiro MLG, Silva HV, Dantas MO, Costa FGP, Oliveira SF, Jordão Filho J, Silva EL. Exigências nutricionais de lisina para codornas durante a fase de postura, em função do nível de proteína da ração. Revista Brasileira de Zootecnia 2003;32(1):156-161.

Rizzo PV, Guandolini GC, Amoroso L, Malheiros RD, Moraes VMB Triptofano na alimentação de codornas japonesas nas fases de recria e Postura. Revista Brasileira de Zootecnia 2008:37(6):1017-1022.

Silva JHV, Oliveira JNC, Silva EL, Jordão Filho J, Ribeiro MLG. Uso da farinha integral da vagem de algaroba (Prosopis juliflora (Sw.) D.C.) na alimentação de codornas japonesas. Revista Brasileira de Zootecnia 2002:31(4):1789-1794.

Silva JHV, Silva MB, Silva EL, Jordão Filho J, Ribeiro MLG, Costa FGP, Dutra Júnior WM. Energia metabolizável de ingredientes determinada com codornas japonesas (Coturnix coturnix japonica). Revista Brasileira de Zootecnia 2003;32(6):1912-1918.

Soares MB, Fuentes MFF, Freitas ER, Lopes IRV, Moreira RF, Sucupira FS, Braz NM, Lima RC. Farelo de amêndoa da castanha de caju na alimentação de codornas japonesas na fase de postura. Revista Brasileira de Zootecnia 2007;36(4):1076-1082

Tukey JW. Exploratory data analysis. Reading: Addison-Wesley; 1977

Umigi RT, Barreto SLT, Donzele JL, Reis RS, Sousa MF, Leite CDS. Níveis de treonina digestível em dietas para codorna japonesa em postura. Revista Brasileira de Zootecnia 2007:36(6):1868-1874. 